\title{
Reproductive dysfunction in women with epilepsy: recommendations for evaluation and management
}

\author{
J Bauer, J I T Isojärvi, A G Herzog, M Reuber, D Polson, E Taubøll, P Genton, \\ $H$ van der Ven, B Roesing, G J Luef, C A Galimberti, J van Parys, D Flügel, \\ A Bergmann, C E Elger
}

See end of article for authors' affiliations

Correspondence to: DrJ Baver,

Universitätsklinikum Bonn

Klinik für Epileptologie,

Sigmund Freud Str 25,

D-53105 Bonn, Germany;

juergen.baver@

ukb.uni-bonn.de

Received

28 November 2001

In revised form 15 March

2002

Accepted 28 March 2002
Background: Epilepsy is commonly associated with reproductive endocrine disorders. These include polycystic ovary syndrome (PCOS), isolated components of this syndrome such as polycystic ovaries, hyperandrogenaemia, hypothalamic amenorrhoea, and functional hyperprolactinaemia.

Objective: To summarise the currently known relations between epilepsy and reproductive endocrine disorders.

Methods: A review of clinical experience and published reports.

Results: The most likely explanations for endocrine disorders related to epilepsy or antiepileptic drugs are: (1) a direct influence of the epileptogenic lesion, epilepsy, or antiepileptic drugs on the endocrine control centres in the brain; (2) the effects of antiepileptic drugs on peripheral endocrine glands; (3) the effects of antiepileptic drugs on the metabolism of hormones and binding proteins; and (4) secondary endocrine complications of antiepileptic drug related weight changes or changes of insulin sensitivity. Regular monitoring of reproductive function at visits is recommended, including questioning about menstrual disorders, fertility, weight, hirsutism, and galactorrhoea. Particular attention should be paid to patients on valproate and obese patients or those experiencing significant weight gain. Single abnormal laboratory or imaging findings without symptoms may not constitute a clinically relevant endocrine disorder. However, patients with these kinds of abnormalities should be monitored to detect the possible development of a symptomatic disorder associated with, for example, menstrual disorders or fertility problems.

Conclusions: If a reproductive endocrine disorder is found, antiepileptic drug treatment should be reviewed to ensure that it is correct for the particular seizure type and that it is not contributing to the endocrine problem. The possible benefits of a change in treatment must be balanced against seizure control and the cumulative side effect of alternative agents.
$\mathrm{T}$ he first aim in the treatment of patients with epilepsy is the suppression of seizures. Epilepsy can, however, also be associated with other pathological changes which may require investigation and treatment. Such changes include endocrine disorders, and in particular those affecting the reproductive system in women. ${ }^{1-4}$

Reproductive endocrine disorders described in this context include polycystic ovary syndrome, hypothalamic amenorrhoea, premature ovarian failure, and functional hyperprolactinaemia. ${ }^{5}$ The recognition of reproductive endocrine disorders in women with epilepsy is important because they may have serious long term consequences and may be treatable. These disorders contribute to the unusually high rate of infertility among women with epilepsy. ${ }^{6-8}$ Reproductive endocrine disorders, such as polycystic ovary syndrome (PCOS), may also be associated with higher rates of migraine, emotional disorders, and female malignancies. It has been suggested, moreover, that anovulatory cycles, which are characteristic of reproductive endocrine disorders, may be associated with higher seizure frequencies. ${ }^{910}$

We summarise some of the currently known relations between epilepsy and reproductive endocrine disorders to enable physicians involved in the treatment of women with epilepsy to develop evidence based principles for the investigation and management of endocrine problems.

\section{EVALUATION OF WOMEN WITH EPILEPSY FOR REPRODUCTIVE ENDOCRINE DISORDERS}

The frequent occurrence of reproductive endocrine disorders in women with epilepsy makes it important for the neurologist to recognise characteristic symptoms and signs. The evaluation of reproductive endocrine disorders typically falls into the domain of the endocrinologist and gynaecologist. The investigation of endocrine problems in patients with epilepsy, however, may well require close cooperation between neurologists and endocrinologists or gynaecologists, as these specialists may not have a detailed understanding of the effects of epilepsy or antiepileptic drugs on the endocrine system.

In table 1 we summarise some of the common clinical features of reproductive endocrine disorders. These include menstrual irregularity, infertility, weight gain, hirsutism, and galactorrhoea. In table 2 we list some of the investigations that are commonly carried out to diagnose reproductive endocrine disorders. Tests include hormonal measurements, pelvic ultrasonography, and pituitary imaging. We also list the implications of the findings.

Pelvic ultrasonography is indicated if clinical features or hormonal tests raise concern about ovarian pathology. Transvaginal ultrasound is more sensitive than transabdominal ultrasound in the identification of structural abnormalities of the ovaries, including tumours and cystic change. Pituitary magnetic resonance imaging may be indicated if the clinical features (for example, galactorrhoea) or the laboratory results (such as hyperprolactinaemia) suggest an abnormality of the hypothalamic-pituitary axis. However, a small pituitary lactotroph adenoma may not be detected if beyond the resolution of magnetic resonance imaging. 
Table 1 Clinical features of reproductive endocrine disorders in women with epilepsy

\begin{tabular}{|c|c|c|c|}
\hline Symptom/sign & Method & Abnormal findings & Comment \\
\hline Menstrual irregularity & $\begin{array}{l}\text { Menstrual chart for at least } 6 \\
\text { months }\end{array}$ & \multirow{2}{*}{$\begin{array}{l}\text { - }<23 \text { days: polymenorrhoea } \\
\text { - >35 days: oligomenorrhoea } \\
\text { - No bleeding >6 months: amenorrhoea } \\
\text { Inability to conceive after more than } 12 \\
\text { months of regular unprotected intercourse } \\
\text { and exclusion of male causes }\end{array}$} & $\begin{array}{l}\text { Look for other symptoms of endocrine disorder } \\
\text { including thyroid dysfunction; investigate or refer for } \\
\text { investigation }\end{array}$ \\
\hline Infertility & Clinical history & & $\begin{array}{l}\text { Assess menstrual regularity. Endocrinologist and/or } \\
\text { gynaecologist should be consulted to exclude } \\
\text { endocrine disorders such as PCOS, hypothalamic } \\
\text { amenorrhoea, hyperprolactinaemia, thyroid } \\
\text { dysfunction }\end{array}$ \\
\hline \multirow[t]{2}{*}{ Obesity and weight gain } & BMI (weight $(\mathrm{kg}) /$ height $^{2}(\mathrm{~cm})$ & \multirow{2}{*}{$\begin{array}{l}\text { - Obese: } \mathrm{BMI}>25 \\
\text { - Significant weight gain: }>5 \mathrm{~kg} \\
\text { Truncal obesity: WHR }>0.9\end{array}$} & $\begin{array}{l}\text { Assess menstrual regularity. In case of cycle } \\
\text { disturbance: investigate or refer }\end{array}$ \\
\hline & $\begin{array}{l}\text { WHR: ratio of supine } \\
\text { circumference of waist/hips }\end{array}$ & & $\begin{array}{l}\text { Assess menstrual regularity. In case of cycle } \\
\text { disturbance: investigate or refer }\end{array}$ \\
\hline Hirsutism & $\begin{array}{l}\text { Inspection or } \\
\text { Ferriman-Gallwey score }\end{array}$ & Male escutcheon & $\begin{array}{l}\text { May be genetic or ethnic. Assess menstrual regularity. } \\
\text { In case of cycle disturbance: investigate or refer }\end{array}$ \\
\hline Galactorrhoea & History & $\begin{array}{l}\text { Crusting on nipples; expression of breast } \\
\text { milk in non-lactating women }\end{array}$ & $\begin{array}{l}\text { Assess menstrual regularity, look for hirsutism, signs } \\
\text { of hypothyroidism. Investigate or refer }\end{array}$ \\
\hline
\end{tabular}

Table 2 Investigation of women with epilepsy and symptoms or signs of reproductive endocrine disorder

\begin{tabular}{|c|c|c|c|}
\hline Test & Method & Abnormal findings & Comment \\
\hline \multirow[t]{3}{*}{$\mathrm{LH}, \mathrm{FSH}$} & $\begin{array}{l}\text { Measurement of serum levels } \\
\text { (calculation based on an average } \\
\text { of three estimations taken } 20 \\
\text { minutes apart between day } 3 \text { and } \\
6 \text { of the cycle) }\end{array}$ & LH/FSH ratio $>2$ & Suggestive of PCOS \\
\hline & & $\mathrm{FSH}>35 \mathrm{IU} / \mathrm{l} ; \mathrm{LH}>11 \mathrm{IU} / \mathrm{I}$ & Suggestive of menopause \\
\hline & & $\mathrm{LH}<7 \mathrm{IU} / \mathrm{ml}$ & Suggestive of hypothalamic amenorrhoea \\
\hline Prolactin & $\begin{array}{l}\text { Measurement of morning resting } \\
\text { serum levels (not postictal!) }\end{array}$ & $>20 \mu \mathrm{g} / \mathrm{l}$ & $\begin{array}{l}\text { May be mildly raised in patients with epilepsy; rule out } \\
\text { hypothyroidism or pituitary tumour; drugs may have } \\
\text { impact on PRL levels }\end{array}$ \\
\hline Progesterone & $\begin{array}{l}\text { Measurement of serum level (blood } \\
\text { taken during mid-luteal phase } \\
\text { according to menstrual cycle) }\end{array}$ & $<6 \mathrm{nmol} / \mathrm{l}$ & $\begin{array}{l}\text { Low levels indicate anovulation; common cause: PCOS, } \\
\text { HA, HPRL }\end{array}$ \\
\hline \multirow[t]{2}{*}{ Testosterone } & $\begin{array}{l}\text { Measurement of serum level day } \\
3-6 \text { of the cycle }\end{array}$ & $>2.5 \mathrm{nmol} / \mathrm{l}$ & $\begin{array}{l}\text { Common cause: PCOS, valproate; non-classical adrena } \\
\text { hyperplasia may cause modest elevation of testosterone }\end{array}$ \\
\hline & & $>4.0 \mathrm{nmol} / \mathrm{l}$ & Rule out adrenal/ovarian tumour \\
\hline Androstenedione & Measurement of serum level & $>10.0 \mathrm{nmol} / \mathrm{l}$ & Rule out non-classical congenital adrenal hyperplasia \\
\hline DHEAS & Measurement of serum level & $\begin{array}{l}\text { Age } 20-29>3800 \mathrm{ng} / \mathrm{ml} \\
\text { Age } 30-39>2700 \mathrm{ng} / \mathrm{ml}\end{array}$ & Rule out non-classical adrenal hyperplasia \\
\hline \multirow[t]{2}{*}{ Glucose/insulin } & Fasting, morning levels; & Fasting glucose $>7.8 \mathrm{mmol} / \mathrm{I}$ & Suggestive of diabetes \\
\hline & glucose/insulin ratio & Glucose/insulin ratio $>4$ & $\begin{array}{l}\text { Suggestive of reduced insulin sensitivity; associated with } \\
\text { obesity and PCOS }\end{array}$ \\
\hline \multirow[t]{2}{*}{ Pelvic ultrasound } & $\begin{array}{l}\text { Transvaginal or transabdominal } \\
\text { (day } 3 \text { to } 9 \text { of the cycle) }\end{array}$ & $\begin{array}{l}>10 \text { peripheral cysts, } 2-8 \mathrm{~mm} \\
\text { diameter in one ultrasound plane, } \\
\text { thickening of ovarian stroma }\end{array}$ & Polycystic ovaries; associated with PCOS \\
\hline & & $\begin{array}{l}\text { Other structural abnormalities of } \\
\text { ovaries }\end{array}$ & Tumours, atrophy, multifollicular ovaries, etc \\
\hline
\end{tabular}

Exact values and units of measurement may vary from laboratory to laboratory.

FSH, follicle stimulating hormone; DHEAS, dehydroepiandrosterone sulphate; HA, hypophyseal adenoma; HPRL, hyperprolactinaemia; LH, luteinising hormone; PCOS, polycystic ovary syndrome

\section{REPRODUCTIVE ENDOCRINE DISORDERS IN WOMEN WITH EPILEPSY}

PCOS is a common cause of irregular periods in women. It is considered to affect approximately $4-6 \%$ of women in the general population. ${ }^{11}{ }^{12}$ The prevalence of PCOS in patients with temporal lobe epilepsy has been found to be between 10\% and $25 \%$ even if they were not receiving antiepileptic drugs. ${ }^{13}$

PCOS is a form of hyperandrogenic chronic anovulation. Anovulation may be indicated by low mid-luteal phase progesterone levels. The pathogenesis of PCOS involves the acceleration of pulsatile gonadotropin releasing hormone (GnRH) secretion, insulin resistance, hyperinsulinaemia, and downstream metabolic dysregulation. Abnormalities of the reproductive axis are manifested as hypersecretion of luteinising hormone, ovarian theca stromal cell hyperactivity, and hypofunction of the follicle stimulating hormone (FSH)granulosa cell axis resulting in hyperandrogenism, hirsutism, follicular arrest, and ovarian acyclicity. ${ }^{14}$
PCOS should not be confused with isolated polycystic ovaries (polycystic change without symptoms, pathological signs, or hormonal abnormality). Isolated polycystic ovaries are observed in $17-22 \%$ of women in the general population, although higher frequencies have been reported. ${ }^{15}{ }^{16}$ In a population based study, polycystic ovaries was detected in $33 \%$ of women investigated (74/224). ${ }^{15}$ Two third of these women had menstrual irregularity and four fifths had at least one feature of PCOS, including menstrual irregularity, acne, hirsutism, obesity, and raised testosterone or luteinising hormone. The large number of women with PCOS in that study might result from a low recruitment rate $(22 \%$ of all women approached) introducing a bias towards women with reproductive endocrine disorders.

The polycystic ovary structure is believed to develop in pubertal women because of a genetic predisposition. ${ }^{17}$ Only some women with polycystic ovaries will develop PCOS with chronic oligomenorrhoea or amenorrhoea associated with increased serum androgen levels. ${ }^{11}$ There are many potential 
triggers for the development of PCOS, and weight gain is one such factor.

Hypothalamic amenorrhoea, also called hypogonadotropic hypogonadism, has been found in $12 \%$ of 50 consecutive women with temporal lobe epilepsy whereas it is estimated to affect only $1.5 \%$ of the general population. ${ }^{1}$ Hypothalamic amenorrhoea is associated with a disturbed secretion of pituitary gonadotropins with low luteinising hormone levels. Hypothalamic amenorrhoea causes amenorrhoea or oligomenorrhoea and infertility in the absence of signs of hyperandrogenaemia.

The menopause may occur earlier in women with epilepsy than in the general female population. In a series of 50 consecutive women with temporolimbic epilepsy, Herzog et al found that two women (4\%) had primary gonadal failure with amenorrhoea and FSH values above $50 \mathrm{mIU} / \mathrm{ml}$ in their third decade of life, as compared with an expected occurrence of about $1 \%$ in the general population. ${ }^{1}$ In a recent study, perimenopause or menopause before 40 years of age was demonstrated in seven of 50 women (14\%) with epilepsy, compared with three of $82(4 \%)$ in a similarly aged normal control group $(\mathrm{p}<0.05){ }^{18}$

The prevalence of functional hyperprolactinaemia may also be increased in women with epilepsy. ${ }^{19}$ Generalised seizures and seizures involving the temporal lobes lead to postictal prolactin elevations. ${ }^{20}$ The same may be true for interictal epileptic activity propagated to the hypothalamus. ${ }^{19}{ }^{21}$ Functional hyperprolactinaemia causes polymenorrhoea, oligomenorrhoea, or amenorrhoea, subfertility, galactorrhoea, and hirsutism

\section{INTERPRETATION OF REPRODUCTIVE DISORDERS IN WOMEN WITH EPILEPSY}

The most likely explanations for endocrine disorders related to epilepsy or antiepileptic drugs are as follows:

- a direct influence of the epileptogenic lesion, epilepsy, or antiepileptic drugs on the endocrine control centres in the brain (the hypothalamic-pituitary axis);

- the effects of antiepileptic drugs on peripheral endocrine glands;

- the effects of antiepileptic drugs on the metabolism of hormones and binding proteins;

- secondary endocrine complications of antiepileptic drug related weight changes or changes of insulin sensitivity.

A direct role for epilepsy in the pathogenesis of reproductive endocrine disorders is suggested by acute changes in serum prolactin and gonadotropin levels following seizures, ${ }^{202}$ a possible relation between the laterality of temporolimbic epileptiform discharges and the specific type of reproductive endocrine disorder (for example, left unilateral temporolimbic epilepsy has been associated with PCOS, right temporolimbic epilepsy with hypothalamic amenorrhoea), ${ }^{123}$ and the normalisation of menstrual cycles after epilepsy surgery. ${ }^{24}$

Antiepileptic drugs have direct effects on peripheral female endocrine glands in animal models. Valproate has been shown to alter steroidogenesis and increase testosterone to oestradiol ratios in porcine ovarian follicles. ${ }^{25}$ Long term use of valproate increased the number of follicular cysts and altered sex steroid hormone levels in rats. ${ }^{26}$ Valproate, but not lamotrigine, increased the number of ovarian follicular cysts in rats. ${ }^{27}$ All of this experimental work was undertaken in non-epileptic animals. It remains to be proven that direct gonadal antiepileptic drug effects are clinically relevant in humans. Isojärvi et al found an increased number of ovarian cysts in women on valproate monotherapy. ${ }^{2-4}$ A normalisation of such polycystic change was observed after discontinuation of valproate. However, there were corresponding improvements of insulin resistance and it is therefore uncertain whether valproate had a direct or an insulin mediated effect on the ovaries. ${ }^{28}$
Antiepileptic drugs may decrease or increase biologically active serum sex hormone levels. Many of the older antiepileptic drugs-including carbamazepine, phenobarbitone (phenobarbital), and phenytoin-induce hepatic cytochrome P450 dependent steroid hormone breakdown and the production of sex hormone binding globulins (SHBG), thereby reducing biologically active sex hormone serum concentrations..$^{29-37}$

Decreases of free serum testosterone levels during carbamazepine treatment owing to the induction of sex hormone binding globulin have been documented.$^{38}$ The free serum testosterone concentration rises when patients are switched from carbamazepine to oxcarbazepine, which causes less hepatic induction. ${ }^{32}$ A reduced rate of PCOS (a condition characterised by a high testosterone) in women treated for epilepsy with enzyme inducers compared with untreated women with epilepsy $(13 \vee 30 \%)$ has been shown. ${ }^{1}$ Other investigators have found that enzyme induction in carbamazepine treated women with epilepsy causes menstrual disturbance characterised by low oestradiol and a low oestradiol/SHBG ratio in 25\% of cases. ${ }^{32}$

Conversely, hepatic enzyme inhibitors can increase biologically active sex hormone levels. In a prospective study an increase in serum androgen concentrations was documented in women with newly diagnosed epilepsy who were started on valproate. ${ }^{39}$ In girls treated with valproate for a mean of two years, higher serum testosterone levels were found than in untreated controls. ${ }^{40}$ Testosterone levels in excess of 2 SD of the mean were found in $38 \%$ of prepubertal, $36 \%$ of pubertal, and $57 \%$ of postpubertal girls on valproate. It should be pointed out that the clinical relevance of a raised total and/or free testosterone level depends on the presence of symptoms of hyperandrogenism such as cycle disturbance, subfertility, male pattern hair loss, hirsutism, or acne. However, an increase in serum total or free testosterone may contribute to altered gonadotropin secretion and lead to manifestations of reproductive endocrine disorders, so that asymptomatic patients with an isolated elevation of total or free testosterone should be kept under endocrine review. ${ }^{41}$

Several antiepileptic drugs may cause weight gain. This adverse effect has been described with valproate, carbamazepine, vigabatrin, and gabapentin. ${ }^{42-46}$ Weight gain and obesity have direct negative effects on many aspects of health and on life expectancy. Weight increase reduces insulin sensitivity and promotes PCOS development in predisposed women who have no previous hormonal abnormality. Thus antiepileptic drug related weight increases could trigger the manifestation of a clinically relevant endocrine disorder. Weight related endocrine problems may be enhanced by the enzyme inhibiting effects of valproate, and masked by enzyme inducers like carbamazepine. Weight reduction after tapering off valproate has been shown to be associated with a normalisation of menstrual cycles and hormonal disturbances. ${ }^{28}$ There are no studies of the effects of weight reduction without change in drug treatment. It should be pointed out that valproate associated endocrine changes have also been observed in the absence of weight gain. ${ }^{34} 40$

There has been intense debate over the existence of an overrepresentation of PCOS or isolated individual constituent components (polycystic ovaries or hyperandrogenism) in women receiving valproate for epilepsy. In a study of 238 women with epilepsy, $45 \%$ of 29 women on valproate monotherapy had menstrual irregularities (amenorrhoea, oligomenorrhoea, prolonged cycles, and irregular menstruation); $60 \%$ of these women also had polycystic ovaries and $30 \%$ had raised serum testosterone concentrations. ${ }^{2}$ In a cross sectional study of 65 women with epilepsy, polycystic ovaries or hyperandrogenaemia were found in 14 of 22 patients taking valproate monotherapy (64\%). ${ }^{3}$ This group comprised women with isolated polycystic ovaries, isolated hyperandrogenaemia, or both. The association of valproate with polycystic ovaries or 
hyperandrogenaemia was supported by a multicentre study including women from Finland, Norway, and the Netherlands. ${ }^{4}$ Endocrine effects were more commonly seen in women beginning valproate before the age of $20 .^{2}$ Discontinuation of valproate treatment led to a reversal of hyperinsulinaemia, hyperandrogenism, dyslipidaemia, and polycystic ovaries in 12 women followed prospectively for one year. ${ }^{28}$ Isojärvi et al linked the finding of polycystic ovaries and hyperandrogenaemia with medication related weight gain, ${ }^{3}$ but they also observed an increased incidence of hormonal disturbances in lean women treated with valproate. They suggested that hyperinsulinism and low serum levels of insulin-like factor binding protein 1 could lead to hyperandrogenism and polycystic ovaries. Murialdo et al found confirmatory evidence for valproate related endocrine effects with polycystic ovaries in $40 \%$ of patients receiving valproate as part of their anticonvulsant polytherapy, versus $13 \%$ in patients not treated with valproate. ${ }^{478}$ They also found raised serum androgen levels and a reduced mid-luteal progesterone surge in valproate as compared with phenobarbitone or carbamazepine treated women as a marker of anovulation. However, two other cross sectional studies in 93 and 43 patients, respectively, failed to confirm an association between menstrual disturbance and a particular antiepileptic drug. ${ }^{49} 50$

\section{MANAGEMENT OF WOMEN WITH EPILEPSY AND REPRODUCTIVE DISORDERS}

The treatment of epilepsy aims for complete seizure control with as few side effects as possible. ${ }^{51}$ The choice of antiepileptic drug is driven by many considerations including proven efficacy for the particular seizure type, tolerability, the personal experience of the physician, and especially how comfortable the physician and the patient feel using a specific agent. In the treatment of women with epilepsy, several additional factors have to be considered. These include the safety of an antiepileptic drug during pregnancy, the compatibility of the antiepileptic drug with hormonal contraception, and the potential impact on reproductive function as outlined above.

The panel does not consider any antiepileptic drug contraindicated for use in women. However, physicians should be aware of reproductive endocrine dysfunction that may occur in women with epilepsy during treatment. This requires regular monitoring of reproductive function at visits, including questioning about menstrual disorders, fertility, weight, hirsutism, and galactorrhoea. Particular attention should be paid to patients treated with valproate and those who are obese or who experience significant weight gain during treatment with antiepileptic drugs. Reproductive dysfunction warrants diagnosis. This often requires the aid of appropriate investigations that may include hormonal tests, pelvic ultrasound, and pituitary imaging. The diagnosis of a reproductive endocrine disorder should then be considered in terms of aetiology and the possible role of potential contributory factors, including epilepsy and antiepileptic drugs. Single abnormal laboratory tests (for example, increased serum testosterone) or imaging findings (such as polycystic ovarian structure) may not constitute a clinically relevant endocrine disorder. Such findings, however, should alert the physician to a greater potential for the development of a clinically relevant disorder and the need for closer clinical and possibly investigational monitoring.

If a reproductive endocrine disorder is found, antiepileptic drug treatment should be reviewed to ensure that it is correct for the particular seizure type and that it is not contributing to the endocrine problem. The possible benefits of a change of antiepileptic drug treatment must be balanced against seizure control and cumulative side effects of alternative agents. Many of the newer antiepileptic drugs have not been studied with regard to (longer term) endocrine reproductive side effects, and little is known about their safety in pregnancy. If a patient is seizure-free on an antiepileptic drug that could be the cause of adverse reproductive endocrine effects, a monotherapy switch is fraught with the risk of seizure relapse, and lower dose combination treatment with the risk of additional side effects. $^{52}$

Although our understanding of the effects of seizures and antiepileptic drugs on reproductive endocrine function remains incomplete, we are able to identify women with epilepsy who have reproductive dysfunction and may benefit from endocrine investigation and therapeutic intervention. Every clinician involved in the management of epilepsy should be aware of the high prevalence and medical significance of reproductive disorders in women with epilepsy.

\section{CONCLUSIONS}

- Reproductive dysfunction and reproductive endocrine disorders are unusually common among women with epilepsy.

- Reproductive disorders probably contribute to the decreased fertility in women with epilepsy.

- Reproductive function should be screened regularly, looking for menstrual disorder, infertility, obesity or weight gain, hirsutism, and galactorrhoea.

- Reproductive dysfunction may require further assessment with endocrine testing, pelvic ultrasound, and pituitary imaging.

- The diagnosis of a reproductive endocrine disorder should be considered in terms of aetiology and potential contributory factors, including epilepsy and antiepileptic drugs (in particular valproate).

- The possible benefits of a change of antiepileptic drug treatment must be balanced against efficacy in terms of seizure control and the side effects of alternative agents.

- The comprehensive management of women with epilepsy includes counselling about reproductive issues that relate to epilepsy and antiepileptic drug use, as well as monitoring of reproductive function.

\section{Authors' affiliations}

J Baver, C E Elger, Department of Epileptology, University of Bonn, Bonn, Germany

J I T Isoiärvi, Department of Neurology, University of Oulu, Finland

A G Herzog, Harvard Neuroendocrine Unit, Beth Israel Deaconess Medical Center, Boston, Massachusetts, USA

M Reuber, Department of Neurology, Leeds Teaching Hospitals, Leeds, UK

D Polson, Department of Gynaecology, Hope Hospital, Manchester, UK E Taubsill, Department of Neurology, Rikshospitalet, University of Oslo, Oslo, Norway

P Genton, Centre Saint Paul, Marseille, France

H van der Ven, B Roesing, Department of Gynaecological

Endocrinology, University of Bonn, Bonn, Germany

G J Luef, Department of Neurology, University of Innsbruck, Innsbruck, Austria

C A Galimberti, Epilepsy Centre, Neurological Institute "C Mondino" Foundation, Pavia, Italy

J van Parys, Epilepsiecentre Kempenhaeghe, Heeze, The Netherlands D Flügel, Department of Neurology, University of Regensburg,

Regensburg, Germany

A Bergmann, Neurologist, Neuburg/Donau, Germany

\section{REFERENCES}

1 Herzog AG, Seibel MM, Schomer DL, et al. Reproductive endocrine disorders in women with partial seizures of temporal lobe origin. Arch Neurol 1986:43:341-6.

2 Isojärvi JIT, Laatikainen TJ, Knip M, et al. Polycystic ovaries and hyperandrogenism in women taking valproate for epilepsy. N Engl J Med 1993;329:1383-8.

3 Isoiärvi JIT, Laatikainen TJ, Knip M, et al. Obesity and endocrine disorders in women taking valproate for epilepsy. Ann Neurol 1996;39:579-84. 
4 Isoiärvi JIT, Taubøll E, Pakarinen AV, et al. Altered ovarian function and cardiovascular risk factors in valproate treated women. Am J Med 2001;111:290-6.

5 Herzog AG. Disorders of reproduction and fertility. In: Engel J, Pedley TA, eds. Epilepsy: a comprehensive textbook. Philadelphia: Lippincott-Raven, 1997:2013-26.

6 Wallace H, Shorvon S, Tallis R. Age-specific incidence and prevalence rates of treated epilepsy in an unselected population of 2,052,922 and age-specific fertility rates of women with epilepsy. Lancet

1998;352:1970-3.

7 Webber MP, Hauser WA, Ottman R, et al. Fertility in persons with epilepsy: 1935-1974. Epilepsia 1986;27:746-52.

8 Olafsson E, Hauser WA, Gudmundsson G. Fertility in patients with epilepsy: a population-based study. Neurology 1998;51:71-3.

9 Bäckstrom T. Epileptic seizures in women related to plasma estrogen and progesterone during the menstrual cycle. Acta Neurol Scand 1975;54:321-47.

10 Herzog AG, Klein P, Ransil BJ. Three patterns of catamenial epilepsy. Epilepsia 1997;38:1082-8.

11 Knochenhauser ES, Key TJ, Kashar-Miller M et al. Prevalence of polycystic ovary syndrome in unselected black and white women of the southeastern United States: a prospective study. J Clin Endocrinol Metab 1998;83:3078-82

12 Herzog AG, Schachter SC. Valproate and the polycystic ovarian syndrome: final thoughts. Epilepsia 2001:42:311-15.

13 Herzog AG, Seibel MM, Schomer D, et al. Temporal lobe epilepsy: an extrahypothalamic pathogenesis for polycystic ovarian syndrome? Neurology 1984;34:1389-93.

14 Yen SSC. Polcystic ovary syndrome (hyperandrogenic chronic anovulation). In: Yen SSC, Jaffe RB, Barbieri RL, eds. Reproductive endocrinology, 4th ed. Philadelphia: WB Saunders, 1999:436-78.

15 Michelmore KF, Balen AH, Dunger DB, et al. Polycystic ovaries and associated biochemical features in young women. Clin Endocrinol 1999;51:779-86.

16 Polson DW, Adams J, Wadsworth J, et al. Polycystic ovaries. A common finding in normal women. Lancet 1988;i:870-2.

17 Balen AH. Pathogenesis of polycystic ovary syndrome - the enigma unravels? Lancet 1999;354:966-7.

18 Klein P, Serje A, Pezzullo JC. Premature ovarian failure in women with epilepsy. Epilepsia 2001:42:1584-9.

19 Bauer J, Stefan H, Schrell U, et al. Serum prolactin concentrations and epilepsy: a study comparing patients in presurgical evaluation with healthy persons and circadian with seizure related variations. Eur Arch Psychiatry Clin Neurosci 1992;241:365-71.

20 Baver J. Epilepsy and prolactin in adults: a clinical review. Epilepsy Res $1996 ; 24: 1-7$

21 Molaie M, Culebras A, Miller M. Effect of interictal epileptiform discharges on nocturnal plasma prolactin concentrations in epileptic patients with complex partial seizures. Epilepsia 1986;27:724-8.

22 Dana-Haeri J, Trimble MR, Oxley J. Prolactin and gonadotropin changes following generalised and partial seizures. J Neurol Neurosurg Psychiatry 1983;46:331-5

23 Herzog AG. A relationship between particular reproductive endocrine disorders and the laterality of epileptiform discharges in women with epilepsy. Neurology 1993;43:1907-10.

24 Baver J, Stoffel-Wagner B, Flügel D, et al. The impact of epilepsy surgery on serum sex hormones and menstrual cycles in female patients. Seizure 2000;9:389-93

25 Gregoraszczuk E, Wojtowicz AK, Taubøll E, et al. Valproate-induced alterations in testosterone, estradiol and progesterone secretion from porcine follicular cells isolated from small- and medium-sized ovarian follicles. Seizure 2000;9:480-5.

26 Taubøll E, Isojärvi JIT, Flinstad Harbo $\mathrm{H}$, et al. Long-term valproate treatment induces changes in ovarian morphology and serum sex steroid hormone levels in female Wistar rats. Seizure 1999:8:490-3.

27 Sveberg Røste L, Taubøll E, Berner A, et al. Valproate, but not lamotrigine, induces ovarian morphological changes in Wistar rats. Exp Toxicol Pathol 2001;52:545-52.
28 Isoiärvi JI, Rättyä J, Myllylä VV, et al. Valproate, lamotrigine, and insulin-mediated risks in women with epilepsy. Ann Neurol 1998;43 446-51.

29 Isojärvi JI. Serum steroid hormones and pituitary function in female epileptic patients during carbamazepine therapy. Epilepsia 1990:31:438-45

30 Isojärvi JIT, Pakarinen AV, Ylipalosaari PJ, et al. Serum hormones in male epileptic patients receiving anticonvulsant medication. Arch Neurol 1990;47:670-6.

31 Isojarvi JI, Pakarinen AJ, Myllylä VV. A prospective study of serum sex hormones during carbamazepine therapy. Epilepsy Res 1991;9:139-44.

32 Isoiärvi JIT, Pakarinen AJ, Rautio A, et al. Sex hormone levels after replacing carbamazepine with oxcarbazepine. Eur J Clin Pharmacol $1995 ; 47: 461-4$

33 Isoiärvi JI, Laatikainen TJ, Pakarinen AJ, et al. Menstrual disorders in women with epilepsy receiving carbamazepine. Epilepsia 1995;36:676-81.

34 Rättyä J, Turkka J, Pakarinan AJ, et al. Reproductive endocrine effects of valproate, carbamazepine, and oxcarbazepine in men with epilepsy. Neurology $2001 ; 56: 31-6$

35 Barrabry JM, Makin HL, Trafford DJ, et al. Effect of anticonvulsants on plasma testosterone and sex hormone binding globulin levels. J Neurol Neurosurg Psychiatry 1978;41:913-14.

36 Victor A, Lundberg PO, Johansson ED. Induction of sex hormone binding globulin by phenytoin. BM 1977;ii:934-5.

37 Beastall GH, Cowan RA, Gray JM, et al. Hormone binding globulins and anticonvulsant therapy. Scott Med J 1985;30:101-5.

38 Stoffel-Wagner B, Baver J, Flügel D, et al. Serum sex hormones are altered in patients with chronic temporal lobe epilepsy receiving anticonvulsant medication. Epilepsia 1998:39:1164-73.

39 Rättyä J, Pakarinen AV, Repo M, et al. Early hormonal changes during valproate or carbamazepine treatment - a 3 months study. Neurology 2001:57:440-1.

40 Vainionpää LK, Rättyä J, Knip M, et al. Valproate-induced hyperandrogenism during pubertal maturation in girls with epilepsy. Ann Neurol 1999:45:444-50

41 Eagleson CA, Gingrich MB, Pastor CL, et al. Polycystic ovarian syndrome: evidence that flutamide restores sensitivity of the gonadotropin-releasing hormone pulse generator to inhibition by estradiol and progesterone. J Clin Endocrinol Met 2000;85:4047-52

42 Biton V, Mirza W, Montouris G, et al. Weight change associated with valproate and lamotrigine monotherapy in patients with epilepsy. Neurology 2001;56:172-7.

43 Breum L, Astrup A, Gram L, et al. Metabolic changes during treatment with valproate in humans: implication for untoward weight gain. Metabolism 1992;41:666-70.

44 Luef G, Abraham I, Trinka E, et al. Weight change associated with valproate and lamotrigine monotherapy in patients with epilepsy. Neurology 2001;57:565-6.

45 Rättyä J, Vainionpaa L, Knip M, et al. The effects of valproate, carbamazepine, and oxcarbazepine on growth and sexual maturation in girls with epilepsy. Pediatrics 1999;3:588-93

46 Pijl H, Meinders AE. Bodyweight change as an adverse effect of drug treatment. Drug Saf 1996;14:329-42.

47 Murialdo G, Galimberti CA, Magri F, et al. Menstrual cycle and ovary alterations in women with epilepsy on antiepileptic drugs. J Endocrinol Invest 1997;20:519-26.

48 Murialdo G, Galimberti CA, Gianelli MV, et al. Effects of valproate, phenobarbital and carbamazepine on sex steroid setup in women with epilepsy. Clin Neuropharmacol 1998;21:52-8.

49 Bauer J, Jarre A, Klingmüller D, et al. Polycystic ovary syndrome in patients with focal epilepsy: a study in 93 women. Epilepsy Res 2000;41:163-7.

50 Luef G, Abraham I, Trinka E, et al. Hyperandrogenism, postprandial hyperinsulism and the risk of PCOS in a cross sectional study of women with epilepsy treated with valproate. Epilepsy Res 2002:48:91-102.

51 Brodie MJ, French JA. Management of epilepsy in adolescents and adults. Lancet 2000;356:323-9.

52 Schmidt D, Gram L. Monotherapy versus polytherapy in epilepsy: a reappraisal. CNS Drugs 1995:3:194-208. 